(C) Masson, Paris, 1979.

\title{
Iconographie des caractères différentiels des larves de Fannia canicularis, F. scalaris et F. manicata
}

par M. KREMER, C. REBHOLTZ et J.-C. DELECOLLE

Laboratcire de Parasitologie de la Faculté de Médecine, 3, rue Kaberlé, F 67000 Strasbourg

\section{Résumé.}

Les auteurs indiquent les caractéristiques des faces ventrale et dorsale des larves de Fannia. Ils précisent sur dessins les caractères morphologiques permettant de reconnaître les espèces scalaris, manicata et canicularis.

\section{Summary.}

Iconography of the differential characteristics of Fannia larvae.

The authors are showing the characteristics of Fannia larvae ventral and dorsal sides. They specify on figures the morphological characters that permit to distinguish the species scalaris, manicata and canicularis.

\section{Origine du matériel}

Dans un échantillon d'urines d'une malade, nous avons déterminé des larves appartenant à trois espèces de Fannia. Les circonstances du prélèvement ne permettent pas de conclure à l'existence d'une myiase urinaire.

En effet, les urines ont été recueillies par la malade elle-même et à son domicile. Or, cette malade de 76 ans vivait dans des conditions d'hygiène très précaire et par

Accepté le 5 février 1979. 
ailleurs, avait un état mental déficient. Une cystite l'amenait à de fréquents et longs séjours à la garde-robe, l'installation sanitaire étant une fosse septique. Un premier prélèvement contenait des $F$. scalaris. Un nouveau prélèvement, fait quelques semai-

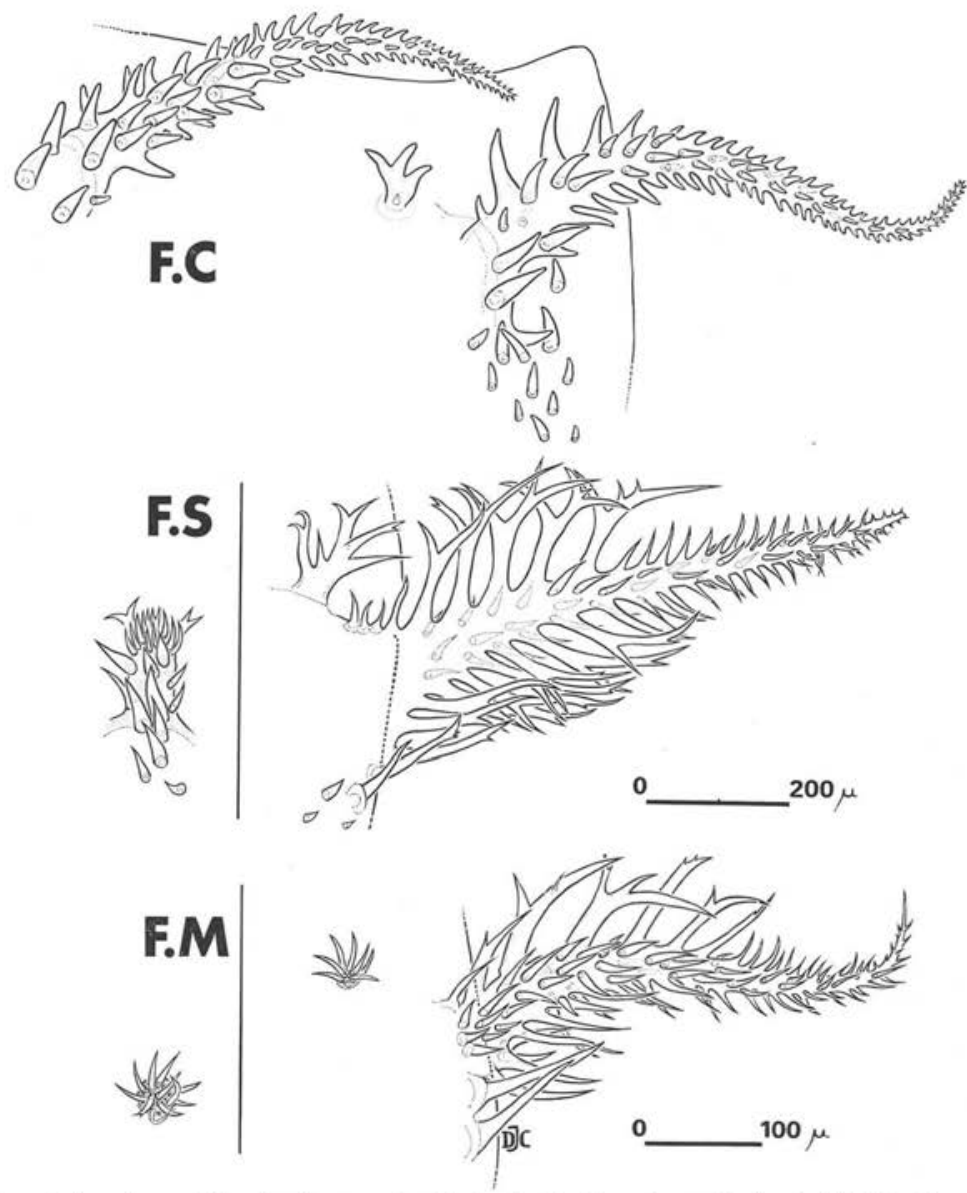

Fig. 1. Avant-dernier article des larves du $3^{\mathrm{e}}$ stade de Fannia canicularis (F.C.), Fannia scalaris (F.S.) et Fannia manicata (F.M.) ; cette dernière a un grossissement différent des deux précédentes. Le processus dorsal de F.C. est en position normale et ceux de F.S. et F.M. ont été rapprochés du processus latéral.

nes plus tard, contenait les trois espèces de Fannia: scalaris (Fabricius), manicata (Meigen) et canicularis (L.) au $3^{\mathrm{e}}$ stade larvaire (fig. 1).

La malade a accepté de se faire hospitaliser pendant 24 heures. Les urines prélevées alors par sondage ne contenaient pas de larves et étaient stériles, une cystoscopie, hélas, n'a pas été pratiquée. L'urographie n'a rien montré de particulier. Peu de temps après, la malade est décédée des suites d'une fracture. 
Du point de vue clinique, cette observation ne présente donc que peu d'intérêt, la myiase urinaire ne pouvant être affirmée avec certitude.

\section{Commentaires sur les caractères morphologiques des larves}

Nous représentons sur les figures ci-contre les processus latéraux et dorsaux de l'avant-dernier segment des larves.

Pour l'identification de celles-ci, nous avons suivi la clef de Zumpt (1965), qui conserve toute sa valeur. Par contre, les illustrations données par cet auteur sont très schématiques et nous pensons utile de les compléter. La morphologie des processus est très riche.

Lorsque les larves ne sont pas montées entre lame et lamelle, il est facile de distinguer la face dorsale de la face ventrale : la première porte les stigmates postérieurs. Lorsque la larve est montée entre lame et lamelle, la situation des stigmates peut être plus difficile à préciser au microscope, en raison de la surimpression des plans, et ceci peut entraîner des confusions entre les processus dorsaux (seuls utilisables dans la clef) et certaines formations ressemblant aux types très réduits des processus dorsaux.

Aussi faut-il préciser que la face dorsale est caractérisée par :

- la présence des stigmates ;

- les processus dorsaux de taille variable et de ce fait utiles dans la clef ;

- les processus latéro-dorsaux comportant un processus accessoire, utile à la reconnaissance du caractère dorsal de ces processus.

La face ventrale est caractérisée par :

- l'absence de stigmates ;

- les processus ventraux au nombre de une ou plusieurs paires, mais toujours réduits à de très petits moignons ;

- les processus latéro-ventraux sans petits processus accessoires.

Ceci est résumé sur la figure 2 .

Fig. 2. Schéma des processus des faces dorsales et ventrales des Fannia.

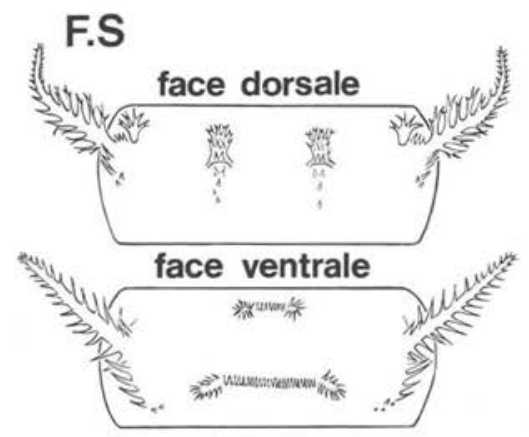


Cette précision concernant la reconnaissance des faces ventrale et dorsale permet d'utiliser la clef de Zumpt sans aucune difficulté.

\section{REMERCIEMENTS}

Les auteurs adressent leurs remerciements aux médecins et biologistes strasbourgeois que ce cas intrigua et qui leur ont fourni ces insectes.

\section{Bibliographie}

Zumpt E. : Myiasis in man and animals in the Old World. Butterworths, édit., Londres, 1965. 